\title{
Universe Filled with Generalized Cosmic Chaplygin Gas and Barotropic Fluid
}

\author{
Kangujam Priyokumar Singh, Rajshekhar Roy Baruah* \\ Department of Mathematical Sciences, Bodoland University, Kokrajhar, India \\ Email: pk_mathematics@yahoo.co.in, "rsroybaruah007@gmail.com
}

Received 11 December 2015; accepted 26 March 2016; published 30 March 2016

Copyright (C) 2016 by authors and Scientific Research Publishing Inc.

This work is licensed under the Creative Commons Attribution International License (CC BY). http://creativecommons.org/licenses/by/4.0/

(c) (i) Open Access

\begin{abstract}
In this paper we have considered a model of the universe filled with Generalized Cosmic Chaplygin Gas and another fluid with barotropic equation of state. We observe its role in accelerating phase of the universe by considering the mixture of these two fluid models valid from the radiation era to $\Lambda \mathrm{CDM}$ for $-1 \leq \gamma \leq 1$ and the radiation era to quintessence model for $\gamma<-1$. The statefinder parameters describe the evolution of the universe in different phases for these two fluid models.
\end{abstract}

\section{Keywords}

Dark Energy, Generalized Cosmic Chaplygin Gas, Barotropic Fluid, Statefinder Parameters, Accelerating Universe

\section{Introduction}

Recent cosmological measurements obtained by SNe Ia [1] [2], WMAP [3], SDSS [4] and X-ray [5] [6] indicate that our universe is expanding and the expansion of the universe is accelerating. The notion known as dark energy (DE) with large negative pressure is proposed to explain this phenomenon which marks the beginning of a new era in cosmology. At present there are a lot of theoretical models of dark energy. Cosmological constant is the simplest model of dark energy corresponding to the $\Lambda \mathrm{CDM}$ model. Besides cosmological constant, the other dark energy models are quintessence [7], phantom [8], tachyon [9], holographic dark energy [10] [11], K-essence [12] and Chaplygin gas models with various equation of state. Our universe consists of about $70 \%$ dark energy, 25\% dark matter and 5\% normal matter (Cold matter and Baryons) and negligible radiation according to the cosmological measurements and analysis. For the accelerated expansion of the universe filled with fluids, the pressure $p$ and the energy density $\rho$ of the universe should violate the strong energy condition $\rho+3 p<0$ i.e., pressure must be negative to accelerate the expansion of the universe.

*Corresponding author.

How to cite this paper: Singh, K.P. and Roy Baruah, R. (2016) Universe Filled with Generalized Cosmic Chaplygin Gas and Barotropic Fluid. International Journal of Astronomy and Astrophysics, 6, 105-110.

http://dx.doi.org/10.4236/ijaa.2016.61008 
In Chaplygin gas (CG) the equation of state parameter for dark energy can be less than -1 as based on the observational data where the equation of state (EoS) [13] is $p=-B / \rho,(B>0)$. The Chaplygin gas is connected to string theory which can be obtained by the Nambu-Goto action moving in a $(d+2)$-dimensional space-time in the light-cone parametrization [14]. The transition period can be described when the universe is filled with Chaplygin gas from a decelerated cosmological expansion to the present exponentially accelerated universe. A viable model [15]-[17] is introduced by generalizing the above equation to the form $p=-B / \rho^{\alpha}$ with $0 \leq \alpha \leq 1$, which is known as generalized Chaplygin gas (GCG), consisting of two free parameters $B$ and $\alpha$ respectively. At high density the generalized Chaplygin gas corresponds to a dust $(p=0)$ universe which is not applicable to our universe. Therefore, modified Chaplygin gas (MCG) was introduced by Benaoum [18] [19] with the equation of state $p=A \rho-B / \rho^{\alpha},(A>0)$. This equation of state describes the evolution of the universe for small values of the cosmological scale factor corresponding to radiation era as well as for large values of the cosmological scale factor corresponding to $\Lambda \mathrm{CDM}$ model. The modified Chaplygin gas may be equivalently described in terms of a homogeneous minimally coupled scalar field $\phi$ in a Friedmann model.

Generalized Cosmic Chaplygin gas [20] [21] was introduced in 2003 by P. F. Gonzalez-Diaz. This model can be made to be stable and free from unphysical behaviors even when the vacuum fluid satisfies the phantom energy condition, which is the striking factor of this model. The equation of state of Generalized Cosmic Chaplygin gas is

$$
p=-\rho^{-\alpha}\left[C+\left(\rho^{1+\alpha}-C\right)^{-\omega}\right]
$$

where $C=\frac{A}{1+\omega}-1$, with $A$ being a constant that can take on both positive and negative values and $-L<\omega<0 ; L$ being a positive definite constant, which can take on values larger than unity.

W. Chakraborty and U. Debnath et al. [22] has obtained the acceleration of the universe containing the modified Chaplygin gas and barotropic fluid. J. K. Singh et al. [23] within the framework of Lyra's geometry studied the modified Chaplygin gas with statefinder parameters. In this paper we try to observe the state of the universe by using Generalized Cosmic Chaplygin gas and barotropic fluid and obtain the values of the statefinder parameters.

\section{Field Equations and Their Solutions}

The metric of a homogenous and isotropic universe in FRW model is

$$
\mathrm{d} s^{2}=\mathrm{d} t^{2}-a^{2}(t)\left[\frac{\mathrm{d} r^{2}}{1-k r^{2}}+r^{2}\left(\mathrm{~d} \theta^{2}+\sin ^{2} \theta \mathrm{d} \phi^{2}\right)\right]
$$

where $a(t)$ is the scale factor and $k(=0, \pm 1)$ is the curvature scalar.

The Einstein field equations are (for $8 \pi G=c=1$ )

$$
\frac{\dot{a}^{2}}{a^{2}}+\frac{k}{a^{2}}=\frac{1}{3} \rho
$$

and

$$
\frac{\ddot{a}}{a}=-\frac{1}{6}(\rho+3 p) .
$$

The energy conservation equation is

$$
\dot{\rho}+3 \frac{\dot{a}}{a}(\rho+p)=0 .
$$

For Generalized Cosmic Chaplygin gas, Equation (5) yields

$$
\rho=\left[C+\left\{1+\frac{B}{a^{3(1+\alpha)(1+\omega)}}\right\}^{\frac{1}{1+\omega}}\right]^{\frac{1}{1+\alpha}}
$$


where $B$ is an arbitrary integration constant.

We consider two fluid cosmological model containing a component of Generalized Cosmic Chaplygin gas, with equation of state (5) and also a component of barotropic fluid with equation of state $p_{1}=\gamma \rho_{1}$. $\omega$ satisfies $-1 \leq \omega \leq 1$ for accelerating universe normally. But $\omega$ satisfies $-1.6 \leq \omega \leq 1$ according to observations i.e., $\omega<-1$ corresponds to phantom model. The right hand side of Equations (3) and (4), i.e. $\rho$ and $p$ should be replaced by $\rho+\rho_{1}$ and $p+p_{1}$ for these two fluid components respectively. We assumed that the two fluids are conserved separately. For Generalized Cosmic Chaplygin gas the expression for the density is given by Equation (6). For another fluid, from Equation (5) the expression for density is given by

$$
\rho_{1}=\frac{d}{a^{3(1+\gamma)}}
$$

where $d$ is an integration constant.

Now to derive the expression for the potential, the following Lagrangian is considered

$$
L_{\phi}=\frac{\dot{\phi}^{2}}{2}-V(\phi) \text {. }
$$

The analogous energy density $\rho_{\phi}$ and pressure $p_{\phi}$ corresponding scalar field $\phi$ having a self interacting potential $V(\phi)$ are given by

$$
\begin{gathered}
\rho_{\phi}=\frac{\dot{\phi}^{2}}{2}+V(\phi)=\rho+\rho_{1}=\left[C+\left\{1+\frac{B}{a^{3(1+\alpha)(1+\omega)}}\right\}^{\frac{1}{1+\omega}}+\frac{d}{a^{3(1+\gamma)}}\right. \\
p_{\phi}=\frac{\dot{\phi}^{2}}{2}-V(\phi)=p+p_{1}=-\left[C+\left\{1+\frac{B}{a^{3(1+\alpha)(1+\omega)}}\right\}^{\frac{1}{1+\omega}}\right]^{-\frac{\alpha}{1+\omega}}\left[C+\left\{1+\frac{B}{a^{3(1+\alpha)(1+\omega)}}\right\}^{-\frac{\omega}{1+\omega}}\right]+\frac{\gamma d}{a^{3(1+\gamma)}} .
\end{gathered}
$$

Now for flat universe $(k=0)$ and assuming $\gamma=C$ we have the expression for $\phi$ and $V(\phi)$ as follows

$$
\begin{gathered}
\phi=\int\left[Y^{\frac{1}{1+\alpha}}-Y^{-\frac{\alpha}{1+\alpha}}\left[C+(Y-C)^{-\omega}\right]+\frac{(1+C) d}{a^{3(1+\gamma)}}\right]^{\frac{1}{2}} \mathrm{~d} t \\
V(\phi)=\frac{1}{2} Y^{\frac{1}{1+\alpha}}+\frac{1}{2} Y^{-\frac{\alpha}{1+\alpha}}\left[C+(Y-C)^{-\omega}\right]+\frac{(1-C) d}{a^{3(1+\gamma)}}
\end{gathered}
$$

where $Y=C+\left\{1+\frac{B}{a^{3(1+\alpha)(1+\omega)}}\right\}^{\frac{1}{1+\omega}}$.

For simplicity we have studied $\gamma=C$ where the two fluids for early universe coincide at higher densities.

\section{The Statefinder Parameters}

It was of utmost necessity to devise a method that would both qualitatively and quantitatively discriminate between various dark energy models, as numerous dark energy models began appearing. Sahni et al. in 2003 proposed a pair of parameters $\{r, s\}$, called statefinder parameters in this context. In a independent manner these parameters are able to discriminate between different dark energy models. The statefinder diagnostic pair has the following form

$$
r=\frac{\dddot{a}}{a H^{3}} \text { and } s=\frac{r-1}{3\left(q-\frac{1}{2}\right)}
$$


where $H\left(=\frac{\dot{a}}{a}\right)$ and $q\left(=\frac{a \ddot{a}}{\dot{a}^{2}}\right)$ are the Hubble parameter and the deceleration parameter respectively. These parameters allow us to characterize the properties of dark energy as these are dimensionless. Corresponding to different cosmological models, trajectories in the $\{r, s\}$ plane corresponds to the fixed point $s=0, \quad r=1$.

These statefinder parameters for one fluid model are given by

$$
\begin{gathered}
r=1+\frac{9}{2}\left(1+\frac{p}{\rho}\right) \frac{\partial p}{\partial \rho} \\
s=\left(1+\frac{\rho}{p}\right) \frac{\partial p}{\partial \rho} .
\end{gathered}
$$

Equations (14) and (15) take the following form for the two fluid components,

$$
\begin{gathered}
r=1+\frac{9}{2\left(\rho+\rho_{1}\right)}\left[\frac{\partial p}{\partial \rho}(\rho+p)+\frac{\partial p_{1}}{\partial \rho_{1}}\left(\rho_{1}+p_{1}\right)\right] \\
s=\frac{1}{\left(p+p_{1}\right)}\left[\frac{\partial p}{\partial \rho}(\rho+p)+\frac{\partial p_{1}}{\partial \rho_{1}}\left(\rho_{1}+p_{1}\right)\right] .
\end{gathered}
$$

The deceleration parameter $q$ has the form

$$
q=-\frac{\ddot{a}}{a H^{2}}=\frac{1}{2}+\frac{3}{2}\left(\frac{p+p_{1}}{\rho+\rho_{1}}\right) .
$$

For Generalized Cosmic Chaplygin gas and barotropic equation of state,

$$
\begin{gathered}
x=\frac{p}{\rho}=-\frac{1}{\rho^{1+\alpha}}\left[C+\left(\rho^{1+\alpha}-C\right)^{-\omega}\right] \\
y=\frac{\rho_{1}}{\rho}=\frac{\frac{d}{a^{3(1+\gamma)}}}{\left[C+\left\{1+\frac{B}{a^{3(1+\alpha)(1+\omega)}}\right\}^{\frac{1}{1+\omega}}\right]^{\frac{1}{1+\alpha}}} .
\end{gathered}
$$

Thus Equations (16) and (17) can be written as

$$
\begin{gathered}
r=1+\frac{9 s}{2}\left(\frac{x+\gamma y}{1+y}\right) \\
s=\frac{(1+x)\left\{\omega(\alpha+1)-x \alpha \rho^{-2 \alpha}\right\}+\gamma(1+\gamma) y}{x+\gamma y} .
\end{gathered}
$$

Now $q<0$ for cosmic acceleration and since $y>0$ we get $x+\gamma<-1 / 3$. The ratio between energy density of the barotropic fluid to that of Generalized Cosmic Chaplygin gas is denoted by $y$ and the ratios of fluid pressure to energy density for barotropic fluid and Generalized Cosmic Chaplygin gas is denoted by $x, \gamma$ respectively. We can assign different values to the barotropic index, since $\gamma$ is constant. But at least one of the fluids must generate negative pressure for cosmic acceleration.

For different values of $\gamma$ we get different cases which are as follows:

Case 1): For $\gamma=1 / 3$, we get $x<-2 / 3$ which corresponds to a universe having cosmic acceleration but unable to violate the strong energy condition as it does not contain barotropic fluid;

Case 2): For $\gamma=0$ and $x<-1 / 3$ it also corresponds to cosmic acceleration and here Chaplygin gas violates the strong energy condition. The barotropic fluid represents the dust;

Case 3): For $\gamma=-1$ we get cosmic acceleration and here the Chaplygin gas does not violate the strong energy condition; 
Case 4): For $\gamma<-1$ the Chaplygin gas represents the dark energy or the dark matter depending upon the values of $x$ and the barotropic fluid represents the phantom model.

\section{Conclusion}

The pair of parameters $(r, s)$ called statefinder parameters distinguish between different types of dark energy. The statefinders were introduced to characterize flat universe models with cold matter (dust) and dark energy. In this paper we have discussed the universe filled with two fluids, Generalized Cosmic Chaplygin gas and barotropic fluid to observe the phases of the universe when it undergoes acceleration using the statefinder parameters and obtain different situations depending upon the values of $\gamma$ where both the fluids represent dark energy and sometimes dark matter. By considering the mixture of the two fluids, we observe the accelerating phase of the universe from the radiation era to $\Lambda \mathrm{CDM}$ for $-1 \leq \gamma \leq 1$ and the radiation era to quintessence model for $\gamma<-1$. When $\gamma=1 / 3$, then $x<-2 / 3$ which corresponds to cosmic acceleration, not violating strong energy condition; when $\gamma=0$, then $x<-1 / 3$ which corresponds to cosmic acceleration, violating strong energy condition. When $\gamma=-1$, then it corresponds to cosmic acceleration where Chaplygin gas does not violate strong energy condition and when $\gamma<-1$, then Chaplygin gas represents dark energy or dark matter and barotropic fluid represents phantom model.

\section{References}

[1] Riess, A.G., et al., Supernova Search Team Collaboration (1998) Observational Evidence from Supernovae for an Accelerating Universe and a Cosmological Constant. The Astrophysical Journal Supplement Series, 116, 1009-1038.

[2] Perlmutter, S.J., et al. (1998) Discovery of a Supernova Explosion at Half the Age of the Universe. Nature, 391, 51-54. http://dx.doi.org/10.1038/34124

[3] Tegmark, M., et al. (2004) Cosmological Parameters from SDSS and WMAP. Physical Review D, 69, Article ID: 103501. http://dx.doi.org/10.1103/PhysRevD.69.103501

[4] Allen, S.W., Schmidt, R.W., Ebeling, H., Fabian, A.C. and Van Speybroeck, L. (2004) Constraints on Dark Energy from Chandra Observations of the Largest Relaxed Galaxy Clusters. Monthly Notices of the Royal Astronomical Society, 353, 457-467. http://dx.doi.org/10.1111/j.1365-2966.2004.08080.x

[5] Spergel, D.N., Verde, L., Peiris, H.V., Komatsu, E., Nolta, M.R., Bennett, C.L., Halpern, M., Hinshaw, G., Jarosik, N., Kogut, A., Limon, M., Meyer, S.S., Page, L., Tucker, G.S., Weiland, J.L., Wollack, E. and Wright, E.L. (2003) First Year Wilkinson Microwave Anisotropy Probe (WMAP) Observations: Determination of Cosmological Parameters. The Astrophysical Journal Supplement Series, 148, 175-194. http://dx.doi.org/10.1086/377226

[6] Komatsu, E., et al. (2009) Five-Year Wilkinson Microwave Anisotropy Probe (WMAP) Observations: Cosmological Interpretation. The Astrophysical Journal Supplement Series, 180, 330-376.

http://dx.doi.org/10.1088/0067-0049/180/2/330

[7] Wetterich, C. (1988) Cosmology and the Fate of Dilatation Symmetry. Nuclear Physics B, 302, 668-696. http://dx.doi.org/10.1016/0550-3213(88)90193-9

[8] Caldwell, R.R. (2002) A Phantom Menace? Cosmological Consequences of Dark Energy Component with Super Negative Equation of State. Physics Letters B, 545, 23-29. http://dx.doi.org/10.1016/S0370-2693(02)02589-3

[9] Setare, M.R., Sadeghi, J. and Amani, A.R. (2009) Interacting Tachyon Dark Energy in Non-Flat Universe. Physics Letters B, 673, 241-246. http://dx.doi.org/10.1016/j.physletb.2009.02.041

[10] Setare, M.R. (2007) Holography Chaplygin Gas Model. Physics Letters B, 648, 329-332. http://dx.doi.org/10.1016/j.physletb.2007.03.025

[11] Setare, M.R. (2009) Holographic Chaplygin DGP Cosmologies. International Journal of Modern Physics D, 18, 419-427. http://dx.doi.org/10.1142/S0218271809014558

[12] Afshordi, N., Chung, D.J. and Geshnizjani, H. (2007) Casual Field Theory with an Infinite Speed of Sound. Physical Review D, 75, Article ID: 083513. http://dx.doi.org/10.1103/PhysRevD.75.083513

[13] Kamenshchik, A., Moschella, U. and Pasquier, V. (2001) An Alternative to Quintessence. Physics Letters B, 511, 265. http://dx.doi.org/10.1016/S0370-2693(01)00571-8

[14] Bordemann, M. and Hoppe, J. (1993) The Dynamics of Relativistic Membranes, Reduction to 2-Dimensional Fluid Dynamics. Physics Letters B, 317, 315-320. http://dx.doi.org/10.1016/0370-2693(93)91002-5

[15] Gorini, V., Kamenshchik, A. and Moschella, U. (2003) Can the Chaplygin Gas Be a Plausible Model for Dark Energy? 
Physical Review D, 67, Article ID: 063509. http://dx.doi.org/10.1103/PhysRevD.67.063509

[16] Alam, U., Sahni, V., Saini, T.D. and Starobinsky, A.A. (2003) Exploring the Expanding Universe and Dark Energy Using the Statefinder Diagnostic. Monthly Notices of the Royal Astronomical Society, 344, 1057-1074. http://dx.doi.org/10.1046/j.1365-8711.2003.06871.x

[17] Bento, M.C., Bertolami, O. and Sen, A.A. (2002) Generalized Chaplygin Gas, Accelerated Expansion and DarkEnergy-Matter Unification. Physical Review D, 66, Article ID: 043507.

http://dx.doi.org/10.1103/PhysRevD.66.043507

[18] Benaoum, H.B. (2002) Accelerated Universe from Modified Chaplygin Gas and Tachyonic Fluid. http://arxiv.org/abs/hep-th/0205140

[19] Benaoum, H.B. (2012) Modified Chaplygin Gas Cosmology. Advances in High Energy Physics, 2012, Article ID: 357802.

[20] Diaz, G. and Pedro, F. (2003) You Need Not Be Afraid of Phantom Energy. Physical Review D, 68, Article ID: 021303 (R). http://dx.doi.org/10.1103/PhysRevD.68.021303

[21] Rudra, P. (2013) Role of Generalized Cosmic Chaplygin Gas in Accelerating Universe: A Field Theoretical Prescription. Modern Physics Letters A, 28, Article ID: 1350102. http://dx.doi.org/10.1142/s0217732313501022

[22] Chakraborty, W. and Debnath, U. (2007) Is Modified Chaplygin Gas with Barotropic Fluid Responsible for Acceleration of the Universe? Modern Physics Letters A, 22, 1805-1812. http://dx.doi.org/10.1142/S021773230702227X

[23] Singh, J.K. and Rani, S. (2015) Modified Chaplygin Gas Cosmology with Statefinder Diagnostic in Lyra's Geometry. Applied Mathematics and Computation, 259, 187-197. http://dx.doi.org/10.1016/j.amc.2015.02.055 